\title{
Multiple Myeloma-Related Peripheral Neuropathy
}

\author{
Haotian Shi ${ }^{1}$ and Fan Zhou ${ }^{1,2 *}$ \\ ${ }^{1}$ Department of Hematology and Oncology, Shanghai Jing'an District Zhabei, Central Hospital, China \\ ${ }^{2}$ Myeloma cooperative group of Shanghai district and county blood alliance, China
}

*Corresponding author: Fan Zhou, Department of Hematology and Oncology, Shanghai Jing'an District Zhabei, Central Hospital,

Myeloma cooperative group of Shanghai district and county blood alliance, China

\begin{tabular}{lll}
\hline ARTICLE INFO & & ABSTRACT \\
\cline { 1 - 1 } $\begin{array}{ll}\text { Received: } \\
\text { Published: July 05, } 2021\end{array}$ & $\begin{array}{l}\text { Abbreviations: MM: Multiple Myeloma; CiPN: Chemotherapy-Induced Peripheral Neu- } \\
\text { ropathy; BiPN: Bortezomib Induced Peripheral Neuropathy; MMPN: Multiple Myeloma } \\
\text { Peripheral Neuropathy }\end{array}$
\end{tabular}

Citation: Haotian Shi, Fan Zhou. Multiple Myeloma-Related Peripheral Neuropathy. Biomed J Sci \& Tech Res 37(1)-2021. BJSTR. MS.ID.005955.

\section{Introduction}

Multiple Myeloma (MM) is a malignant disease characterized by a large number of abnormal proliferations of monoclonal plasma cells in bone marrow. Clonal plasma cells and their secreted $\mathrm{M}$ protein directly infiltrate tissues and organs, leading to various clinical symptoms characterized by anemia, bone pain or osteolytic bone destruction, hypercalcemia, and renal insufficiency. MM is the second most common malignancy in the blood system, mainly occurring in middle-aged and elderly people. Men are significantly more than women. The etiology and pathogenesis of MM are not yet clear. The natural course was 6 to 12 months. In recent years, with the advent of new drugs and the improvement of detection methods, the diagnosis and treatment of MM have been continuously improved and perfected. The survival time of MM patients has been significantly prolonged. At the same time, the incidence rate of severe adverse reactions such as Chemotherapyinduced Peripheral Neuropathy (CiPN) is increasing gradually after treatment, especially for the first-line treatment of MM therapy, the proteasome inhibitor bortezomib, the immunoregulatory drug, thalidomide. It is reported that the incidence of Bortezomib induced Peripheral Neuropathy (BiPN) is 40\% 60\% [1-5], and thalidomide induced peripheral neuropathy (TipN) is $25 \% \sim 75 \%$ [6].
The specific pathophysiological mechanism of MM chemotherapy related PN is still unclear, according to the current studies, and the pathogenesis of PN caused by different therapeutic drugs is also different [7]. Because of the difficulty of diagnosis and the complexity of differentiation of PN and the difference of autonomic sensation, the general discussion focuses on sensory, motor, and sensorimotor neuropathy. This article reviews the characteristics and treatment of PN in MM. The etiology of peripheral neuropathy caused by multiple myeloma is as follows

\section{Caused by the Disease Itself}

There are $1-2 \% \sim 20 \%$ of multiple myeloma can lead to peripheral neuropathy [5]. Although the exact cause of PN caused by MM is unknown, amyloid deposition, immunoglobulin M antibody acting on myelin associated glycoprotein, nerve glycoconjugates participating in the interaction between Schwann cells and axons, and cytokine damage is considered to be the possible mechanism of PN. In addition to the form of nervous system complications, the direct compression of tumor can also lead to PN [8-10]. The etiological mechanism of PN is complex, such as small fiber injury, segmental demyelination, and axonal degeneration. PN is also related to monoclonal gamma globulin imbalance, amyloidosis, and 
POEMS syndrome $[8,11]$. The PN pathology of MM is different from other albuminemia, which demonstrates the importance of MM management.

\section{Induced by the Drug}

Reported by the literature, the incidence rate of CiPN is increasing year by year. Because bortezomib and thalidomide are effective in treating MM, and they are cost-effective. At present, they are the main first-line drugs for the treatment of MM, while they both can cause PN. BiPN is mainly sensory type, and there is also mixed type (sensory and motor PN). It is usually characterized by distal sensory abnormalities, burning numbness and neuropathic pain $[12,13]$. BiPN is related to dose, administration time and administration mode, and is usually reversible. The pathological mechanism of BiPN includes:

a) It acts on the cytoplasm of dorsal root ganglion to produce toxic effects, leading to metabolic changes

b) It can promote the release of calcium in endoplasmic reticulum and induce mitochondria to regulate cell apoptosis

c) Bortezomib can inhibit the transcription of nerve growth factor and genetic factor at the same time [14].

After 12 months of treatment, the incidence of TiPN is 70\%, and its pathogenesis is still unclear. The possible related factors are as follows:

a) Thalidomide can affect the axons of sensory nerve and motor nerve, causing neurotoxicity, especially the myelinated long nerve fibers

b) Anti-angiogenesis, which reduces the formation of capillaries and leads to ischemia and hypoxia of nerve fibers

c) The down-regulation of tumor necrosis factor $\alpha$ decreased the survival of neural cells

d) Genetic factors, such as some gene mutations $[15,16]$.

\section{Other factors of Multiple Myeloma Led to PN}

There are few reports on the mechanism of PN induced by other neurotoxic drugs in MM. Clinical data show that vincristine causes distal mutation through local neuroaxonal toxicity [17]. This may be the combination of vincristine and tubulin to interfere with micro polymerization. Platinum induced PN is caused by direct toxic damage to DRG, but it can also involve peripheral and central sensory neuron degeneration [18]. The incidence rate and clinical features of peripheral neuropathy

Symptom: The symptoms of PN are usually symmetrical, including paresthesia, numbness and burning sensation; These symptoms are usually mild and mild, but in rare cases they can be paralyzed or even life-threatening. Although there are some differences in the treatment of $\mathrm{PN}$, the acute symptoms are usually symmetrical, starting from the distal end and gradually aggravating. BiPN is mild and generally tolerable (although it is often reported that more than $15 \%$ of patients have severe sensory and motor PN) [19]. Symptoms generally start from the distal end of the nerve, including burning sensation, hypersensitivity, Hypoesthesia, paresthesia, discomfort, neuropathic pain. Thalidomide induced nerve damage may be permanent, and it may be the symptom of peripheral neuropathy after stopping treatment [20]. It is reported that thalidomide can cause neuropathy of sensory and sensorimotor axons, which usually affects the sensory numbness or pain of the distal end of the foot, and sometimes affects the hand $[21,22]$. The neurotoxicity of vincristine can progress to a distal symmetric sensory neuropathy [23]. Although there is no clear evidence, cisplatin can cause long-term peripheral sensory nerve damage in the treatment of epithelial ovarian cancer [24].

Incidence Rate: A multicenter phase 2 study mainly evaluated the effect of BiPN on MM patients with bortezomib as the firstline treatment. The study showed that $64 \%$ of patients developed acute BiPN, including $3 \%$ of patients with grade 3 neuropathy. In a phase 3 clinical trial of bortezomib based combination regimen, more than $70 \%$ of patients developed BiPN, more than $16 \%$ of the patients had grade $>3$ neuropathy [25-35]. The incidence of BiPN in relapsed and newly diagnosed myeloma patients was basically the same [36-39]. Importantly, the occurrence of BiPN did not affect the therapeutic effect of bortezomib.

The incidence of TiPN is similar to that of BiPN. After treatment for more than 12 months, over $75 \%$ of patients developed peripheral neuropathy [40]. In the naive MM patients, the overall incidence rate, and the incidence of $>3 \mathrm{PN}$ were $55 \%$ and $10 \%$ respectively. For the treatment of relapsed myeloma with thalidomide and thalidomide combined with dexamethasone, $22 \%$ of the patients had $1 / 2$ grade TiPN. $6 \%$ of patients had grade $3 / 4$ TiPN. Compared with thalidomide, lenalidomide was reported to have less incidence of peripheral neuropathy and less peripheral neurotoxicity.

Reversibility: Although PN can reduce the quality of life of patients, BiPN is reversible for most patients. For example, in the VISTA trial, $60 \%$ of patients with peripheral neuropathy disappeared after 5.7 months. It has also been reported that in patients with bone marrow transplantation the induced BiPN by bortezomib, thalidomide and dexamethasone is reversible. The reversibility of BiPN was confirmed by neurophysiology and histology in mice $[41,42]$. The reversibility of peripheral neuropathy is very important for bortezomib treatment. Thalidomide induced nerve injury may be permanent, and some research reports point out that TiPN can only be improved slowly or even can't be improved.

\section{Diagnosis of Peripheral Neuropathy (PN)}

At present, the diagnostic criteria of Multiple Myeloma Peripheral Neuropathy (MMPN) are based on the following three aspects: 
a) Definite disease history

b) The corresponding clinical symptoms and signs appeared during or after the diagnosis or treatment

c) Any one or more of the following four aspects of nervous system examination are abnormal: sensory nerve examination; Motor nerve examination; Autonomic nerve examination; One or more items of nerve conduction velocity examination (including sensory nerve and motor nerve conduction velocity measurement) slowed down [43].

Because the symptoms of NP are subjective manifestations of patients, because there are different scales, how to accurately grade $\mathrm{PN}$ is still a controversial issue. We hope to have a grading standard including clinical symptoms and electrophysiology. Clinical examination and electrophysiological measurement can objectively and effectively evaluate the toxic effects of chemotherapy drugs on peripheral nerve injury, but in practice, the measurement of electrophysiology is not easy to achieve. At present, we can only

Table 1: FACT/GOG - Ntx neurotoxicity assessment tool. use EMG to measure electrophysiology. Considering that the total score of neuropathy (TNS) does not include the evaluation of pain intensity, TNS and 11 point pain intensity scale (PI-NRS) may be the best method in the evaluation and grading of PN.

\section{Evaluation of Peripheral Neuropathy}

The symptoms of CiPN are different in patients, and the previous diagnosis and evaluation mainly depend on the subjective reports of patients. Nowadays more and more medical staff have raised their concern about CiPN. However, it is usually only subjective assessment of CiPN symptoms of patients, it is inevitable that the severity and incidence rate of CiPN can be inaccurate. It is necessary to combine CiPN's subjective feelings with objective indicators to evaluate the value of the CiPN. Combined with electrophysiological examination, quality of life questionnaire (FACT/GOG-Ntx) and simple pain assessment questionnaire (NRS and VAS pain score), it can provide an objective and powerful evidence for the incidence rate, clinical symptoms, and pathological classification of CiPN [4447] (Table 1).

\begin{tabular}{|c|c|c|c|c|c|}
\hline Symptom & \multicolumn{5}{|c|}{ Grade } \\
\hline Numbness or tingling in the hand & 0 & 1 & 2 & 3 & 4 \\
\hline Numbness or tingling in feet and hands & 0 & 1 & 2 & 3 & 4 \\
\hline Feel uncomfortable with hands & 0 & 1 & 2 & 3 & 4 \\
\hline Feel uncomfortable with feet & 0 & 1 & 2 & 3 & 4 \\
\hline Arthralgia or muscle spasm & 0 & 1 & 2 & 3 & 4 \\
\hline Feel weak & 0 & 1 & 2 & 3 & 4 \\
\hline Hearing difficulty & 0 & 1 & 2 & 3 & 4 \\
\hline Tinnitus & 0 & 1 & 2 & 3 & 4 \\
\hline Difficult to button & 0 & 1 & 2 & 3 & 4 \\
\hline Can't tell the shape of a small object in your hand & 0 & 1 & 2 & 3 & 4 \\
\hline Walking difficulties & 0 & 1 & 2 & 3 & 4 \\
\hline \multicolumn{6}{|c|}{$0=$ Not at all $1=$ Slight $2=$ Obvious $3=$ Quite Obvious $4=$ Very Serious } \\
\hline \multicolumn{6}{|c|}{ VAS Pain Scoring Criteria (Score $0-10$ ) } \\
\hline 0 & \multicolumn{5}{|c|}{ It doesn't hurt at all } \\
\hline Less than 3 points & \multicolumn{5}{|c|}{ Its painful, while its tolerable } \\
\hline 4-6 points & \multicolumn{5}{|c|}{ Patients with pain and affect sleep, but still can tolerate } \\
\hline 7-10 points & \multicolumn{5}{|c|}{ Patients have gradually strong pain, pain unbearable, affect appetite, affect sleep } \\
\hline
\end{tabular}

\section{Clinical Advice}

The occurrence of PN is very common in MM patients. Recent discussions suggest that there is lack of effective treatment for PN in MM patients, so in order to improve the quality of life of patients and better optimize the effect of chemotherapy, prevention is the key. All MM patients receiving neurotoxic drug chemotherapy should be evaluated before the symptoms of PN appear. The dosage adjustment is the "gold standard" to improve the symptoms of BiPN and TiPN.

\section{The Treatment of PN}

Treatment of Primary Diseases: The control of primary disease is the key to the treatment of PN. PN caused by chemotherapy drugs is usually related to the dose. Once PN occurs, in addition to symptomatic treatment, it is also necessary to immediately adjust the chemotherapy drugs or extend the treatment period. The nerve damage caused by BiPN is reversible. In case of PN, in addition to reducing the dose of bortezomib, it can also be changed from twice a week to once a week by changing the frequency of use or change 
bortezomib to subcutaneous embedding administration to reduce the incidence and degree of PN. In addition, the combined use of bortezomib and dexamethasone can reduce severe neurological complications $[48,49]$. The dose and duration of thalidomide treatment are important factors leading to CiPN. Different from BiPN, current clinical trials have proved that TiPN is basically irreversible when the therapeutic dose of thalidomide accumulates to a certain extent. Therefore, when clinical symptoms of TiPN appear, it is necessary to reduce the dose of thalidomide or even stop using it.

Other Symptomatic Treatment: We should be very careful when using neurotoxic drugs because long-term use of neurotoxic drugs can lead to permanent peripheral nerve injury. It is suggested that patients should take exercise and physical therapy as adjuvant treatment, such as wearing loose clothes, warm water foot bath, etc. Second, Choose neuroprotective agents such as vitamin B, Mecobalamin, glutamine, acetyl L-carnitine, antioxidant (docosahexaenoic acid, $\alpha$ - Lipoic acid, glutathione) etc, in order to repair the pathological damage of nerve as far as possible, to reduce the clinical symptoms of PN. For patients with peripheral neuropathic pain, the recommended treatment sequence is as follows:

\section{a) First-line medication: gabapentin, pregabalin antidepressants}

b) Second line medication: tramadol, oxycodone, opioids, using multi-step analgesic treatment

c) Third line drugs: antiepileptic drugs (Ethylsuccinate), ketamine (some patients can be used as second-line drugs) [43].

In addition, Chinese scholars have found that acupuncture can effectively improve the symptoms of PN, especially the feeling of hand and foot acupuncture, numbness, and discomfort. Acupuncture combined with Mecobalamin has better curative effect than Mecobalamin alone [50,51].

\section{Discussion}

Chemotherapeutic drugs increase the survival rate of $\mathrm{MM}$ but the PN caused by it is also more common. It has become the main adverse reaction and new challenge of drug treatment of MM. Besides, PN is related to primary diseases and chemotherapy drugs, and it is also related to the risk factors such as diabetes, vascular disease, obesity and so on. When we first visit MM, we need to comprehensively evaluate $\mathrm{PN}$, and develop a chemotherapy regimen with optimal safety during chemotherapy. We should prevent and early identify some related manifestations of PN. We should achieve early occurrence, early diagnosis and early treatment, including reducing drug dose, extending drug interval, replacing chemotherapy drugs, or using some neuroprotective agents as soon as possible, or combining acupuncture, physiotherapy and other traditional Chinese medicine techniques, At present, there is no standard and standardized MMPN evaluation and monitoring management system, which makes MMPN diagnosis and efficacy evaluation lack of standards and treatment methods. The clinical application of neuroprotective strategies and the identification of CiPN risk factors based on genotype analysis will provide us with more treatment options in the future [52].

\section{Acknowledgement}

This article is supported by Shanghai three-year action plan project of major clinical research of traditional Chinese Medicine.

\section{References}

1. Dimopoulos MA, Mateos MV, Richardson PG (2011) Risk factors for, and reversibility of, peripheral neuropathy associated with bortezomibmelphalan prednisone in newly diagnosed patients with multiple myeloma: subanalysis of the phase 3 VISTA study. European Journal of Haematology 86(1): 23-31.

2. Richardson PG, Sonneveld P, Schuster MW (2009) Reversibility of symptomatic peripheral neuropathy with boaezomib in the phase III APEX trial in relapsed multiple myeloma impact of a dose-modification guideline. Br J Haematol 144(6): 895-903.

3. Hrusovsky l, Emmerich B, Von Rohr A (2010) Borlezomih retreatment in relapsed multiple myeloma results from a retrospective muhicenter survey in Germany and Switzerland. Oncology 79(3-4): 247-254.

4. Petrneci MT, Giraldo P, Cerradini P (2013) A prospective, international phase 2 study of bortezomib retreatment in patients with relapsed multiple myeloma. Br J Haematol 160(5): 649-659.

5. Richardson PG, Xie W, Mitsiades C (2009) Single-agent bortezomib in previously Untreated multiple myeloma:efficacy, characterization of peripheral neuropathy, and molecular correlations with response and neuropathy. J Clin Oncol 27(21): 3518-3525.

6. Morawska M, Grzasko N, Kostyra M (2015) Therapy-related peripheral neuropathy in multiple myeloma patients. Hematol Oncol 33(4):113119.

7. Marchand F, Perretti M, Mcmahon SB (2005) Role of the immune system in chronic pain. Nat Rev Neurosci 6(7): 521-532.

8. Dispenzieri A, Kyle RA (2005) Neurological aspects of multiple myeloma and related disorders. Best Pract Res Clin Haematol 18(4): 673-688.

9. Ropper AH, Gorson KC (1998) Neuropathies associated with paraproteinemia. N Engl J Med 338: 1601-1607.

10. Tariman JD, Love G, McCullagh E, Sandifer S (2008) Peripheral neuropathy associated with novel therapies in patients with multiple myeloma: consensus statement of the IMF Nurse Leadership Board. Clin J Oncol Nurs 12(3): 29-36.

11. Hausheer FH, Schilsky RL, Bain S, Berghorn EJ, Lieberman F (2006) Diagnosis, management, and evaluation of chemotherapy-induced peripheral neuropathy. Semin Oncol 33(1): 15-49.

12. Delforge M, Blade J, Dimopoulos MA (2010) Treatment-related peripheral neuropathy in multiplemyeloma the challenge continues. Lancet Oncol 11(11): 1086-1095.

13. García Sanz R, Corchete LA, Alcoceba M (2017) Prediction of peripheral neuropathy in multiple myeloma patients receiving bortezomib and thalidomide: a genetic study based on a single nucleotide polymorphism array. Hematol Oncol 35(4): 746-751. 
14. Meng JG, Wang F, Han ZH (2016) The research progress on the mechanism of multiple myeloma with peripheral neuropathy. Medical review 22(24): 4843-4847.

15. Luczkowska K, Litwinska Z (2018) Pathophysiology of drug-induce peripheral neuropathy in patients with multiple myeloma. J Physiol Pharmacol 69(2): 165-172.

16. Mahmoudpour SH, Bandapalli OR, Da Silva Filho MI (2018) Chemotherapy-induced peripheral neuropathy evidence from genomewide association studies and replication within multiple myeloma patients. BMC Cancer 18(1): 820.

17. Silva A, Wang Q Wang M, Ravula SK, Glass JD (2006) Evidence for direct axonal toxicity in vincristine neuropathy. J Peripher Nerv Syst 11(3): 211-216.

18. Krarup Hansen A, Helweg Larsen S, Schmalbruch H, Rorth M, Krarup C (2007) Neuronal involvement in cisplatin neuropathy:prospective clinical and neurophysiological studies. Brain 130(4): 1076-1088.

19. (2011) Millennium Pharmaceuticals Inc. VELCADEOR (bortezomib) for Injection. Prescribing Information. Cambridge, MA, USA, 2011.

20. (2010) Celgene Corporation. THALOMID (thalidomide) Capsules Prescribing Information. Summit, NJ, USA, 2010

21. Mileshkin L, Stark R, Day B, Seymour JF, Zeldis JB (2006) Development of neuropathy in patients with myeloma treated with thalidomide: patterns of occurrence and the role of electrophysiologic monitoring. J Clin Oncol 24(27): 4507-4514.

22. Mileshkin L, Prince HM (2006) The troublesome toxicity of peripheral neuropathy with thalidomide. Leuk Lymphoma 47(11): 2276-2279.

23. Pal PK (1999) Clinical and electrophysiological studies in vincristine induced neuropathy. Electromyogr Clin Neurophysiol 39(6): 323-330.

24. Cavaletti G, Bogliun G, Marzorati L, Tredici G, Colombo N (1994) Longterm peripheral neurotoxicity of cisplatin in patients with successfully treated epithelial ovarian cancer. Anticancer Res 14(38): 1287-1292.

25. Bringhen S, Larocca A, Rossi D, Cavalli M, Genuardi M (2010) Efficacy and safety of once weekly bortezomib in multiple myeloma patients. Blood 116(23): 4745-4753.

26. Cavo M, Tacchetti P, Patriarca F, Petrucci MT, Pantani L (2010) Bortezomib with thalidomide plus dexamethasone compared with thalidomide plus dexamethasone as induction therapy before, and consolidation therapy after, double autologous stemcell transplantation in newly diagnosed multiple myeloma: a randomised phase 3 study. Lancet 376(9758): 2075-2085.

27. Dimopoulos MA, Mateos MV, Richardson PG, Schlag R, Khuageva NK (2011) Risk factors for, and reversibility of, peripheral neuropathy associated with bortezomib-melphalan-prednisone in newly diagnosed patients with multiple myeloma subanalysis of the phase 3 VISTA study. Eur J Haematol 86(1): 23-31.

28. Harousseau JL, Attal M, Avet Loiseau H, Marit G, Caillot D (2010) Bortezomib plus dexamethasone is superior to vincristine plus doxorubicin plus dexamethasone as induction treatment prior to autologous stem-cell transplantation in newly diagnosed multiple myeloma: results of the IFM 2005-01 phase III trial. J Clin Oncol 28(30): 4621-4629.

29. Mateos MV, Oriol A, Martinez Lopez J, Gutierrez N, Teruel AI (2010) Bortezomib, melphalan, and prednisone versus bortezomib, thalidomide, and prednisone as induction therapy followed by maintenance treatment with bortezomib and thalidomide versus bortezomib and prednisone in elderly patients with untreated multiple myeloma: a randomised trial. Lancet Oncol 11(10): 934-941.

30. Palumbo A, Bringhen S, Rossi D, Cavalli M, Larocca A (2010) Bortezomibmelphalan-prednisone-thalidomide followed by maintenance with bortezomib-thalidomide compared with bortezomib-melphalan- prednisone for initial treatment of multiple myeloma: a randomized controlled trial. J Clin Oncol 28(34): 5101- 5109.

31. Rosinol L, Cibeira MT, Mateos MV, Martinez J, Oriol A (2010) A phase III PETHEMA/GEM study of induction therapy prior autologous stem cell transplantation (ASCT) in multiple myeloma: superiority of VTD (bortezomib/thalidomide/dexamethasone) over TD and VBMCP/VBAD plus bortezomib. Blood 116(21): 139-140 .

32. Sonneveld P, Schmidt Wolf I, Van der Holt B, el Jarrari L, Bertsch U (2010) HOVON-65/GMMG-HD4 randomized phase III trial comparing bortezomib, doxorubicin, dexamethasone (PAD) vs VAD followed by high-dose melphalan (HDM) and maintenance with bortezomib or thalidomide in patients with newly diagnosed multiple myeloma (MM). Blood 116(21): 23-24.

33. Zervas K, Mihou D, Katodritou E, Pouli A, Mitsouli CH (2007) VAD-doxil versus VAD-doxil plus thalidomide as initial treatment for multiple myeloma: results of a multicenter randomized trial of the Greek Myeloma Study Group. Ann Oncol 18(8): 1369-1375.

34. Orlowski RZ, Nagler A, Sonneveld P, Blade J, Hajek R (2007) Randomized phase III study of pegylated liposomal doxorubicin plus bortezomib compared with bortezomib alone in relapsed or refractory multiple myeloma: combination therapy improves time to progression. J Clin Oncol 25(25): 3892-3901.

35. Moreau P, Avet Loiseau H, Facon T, Attal M, Tiab M (2011) Bortezomib plus dexamethasone versus reduced-dose bortezomib, thalidomide plus dexamethasone as induction treatment prior to autologous stem cell transplantation in newly diagnosed multiple myeloma. Blood 118(22): 5752-5758.

36. Mikhael JR, Belch AR, Prince HM, Lucio MN, Maiolino A (2009) High response rate to bortezomib with or without dexamethasone in patients with relapsed or refractory multiple myeloma: results of a global phase 3b expanded access program. Br J Haematol 144(2): 169-175.

37. Richardson PG, Briemberg H, Jagannath S, Wen PY, Barlogie B (2006) Frequency, characteristics, and reversibility of peripheral neuropathy during treatment of advanced multiple myeloma with bortezomib. J Clin Oncol 24(19): 3113-3120.

38. Richardson PG, Sonneveld P, Schuster MW, Stadtmauer EA, Facon $\mathrm{T}$ (2009) Reversibility of symptomatic peripheral neuropathy with bortezomib in the phase III APEX trial in relapsed multiple myeloma: impact of a dose-modification guideline. Br J Haematol 144(6): 895-903.

39. Moreau P, Pylypenko H, Grosicki S, Karamanesht I, Leleu X (2011) Subcutaneous versus intravenous administration of bortezomib in patients with relapsed multiple myeloma: a randomised, phase 3 , noninferiority study. Lancet Oncol 12(5): 431-440.

40. Tosi P, Zamagni E, Cellini C, Plasmati R, Cangini D (2005) Neurological toxicity of long-term (41yr) thalidomide therapy in patients with multiple myeloma. Eur J Haematol 74(3): 212-216.

41. Richardson PG, Bruna J, Amato AA, Udina E, Mitsiades CS (2009) Bortezomib-associated peripheral neuropathy relationship between clinical neurophysiologic evidence in previously untreated multiple myeloma patients and preclinical characterization in a mouse model. Blood 114(22): 1485a.

42. Bruna J, Udina E, Ale A, Vilches JJ, Vynckier A (2010) Neurophysiological, histological and immunohistochemical characterization of bortezomibinduced neuropathy in mice. Exp Neurol 223(2): 599-608.

43. (2015) Multiple myeloma Committee of Hematology branch of Chinese Medical Association. The consensus on diagnosis and treatment of peripheral neuropathy in multiple myeloma in China(2015). Chinese Journal of internal medicine 54(9): 603-606.

44. Cavaletti G, Frigeni B, Lanzani F (2010) Chemotherapy-Induced Peripheral Neurotoxicity assessment: a critical revision of the currently available tools. Eur J Cancer 46(3): 479-494. 
45. Bennett BK, Park SB, Lin CS (2012) Impact of oxaliplatin-induced neuropathy: a patient perspective. Support Care Cancer 20(11): 29592967.

46. Group TC (2009) CI-PERINOMS: chemotherapy induced peripheral neuropathy outcome measures study. J Peripher Nerv Syst 14(2): 69-71.

47. Hershman DL, Weimer LH, Wang A (2011) Association between patient reported outcomes and quantitative sensory tests for measuring longterm neurotoxicity in breast cancer survivors treated with adjuvant paclitaxel chemotherapy. Breast Cancer Res Treat 125(3): 767-774.

48. Mangiacavalli S, Cocito F, Ferretti VV (2017) The advantages of switch to subcutaneous bortezomib in the real life. Leuk Lymphoma 59(6): 1-3.

ISSN: 2574-1241

DOI: 10.26717/BJSTR.2021.37.005955

Fan Zhou. Biomed J Sci \& Tech Res

(C) This work is licensed under Creative Commons Attribution 4.0 License

Submission Link: https://biomedres.us/submit-manuscript.php
49. Kumar SK, Laubach JP, Giove TJ (2017) Impact of concomitant dexamethasone dosing schedule on bortezomib-induced peripheral neuropathy in multiple myeloma. Br J Haematol 178(5): 756-763.

50. Zhi WI, Ingram E, Li SQ (2018) Acupuncture for bortezomib-induced peripheral neuropathy not just for pain. Integr Cancer Ther 17(4): 10791086

51. Han XY, Wang LJ, Shi HF (2017) Acupuncture combined with methylcobalamin for the treatment of chemotherapy-induced peripheral neuropathy in patients with multiple myeloma. BMC Cancer 17(1): 40.

52. Koeppen, Susanne (2014) Treatment of multiple myeloma thalidomide bortezomib and lenalidomide-induced peripheral neuropathy. Oncology Research and Treatment 37(9): 506-513.

$\begin{array}{ll}\text { BIOMEDICAL } & \text { Assets of Publishing with us } \\ \text { RESEARCHES } & \text { - Global archiving of articles } \\ & \text { - Immediate, unrestricted online access } \\ & \text { - Rigorous Peer Review Process } \\ & \text { - Anttps://biomedres.us/ }\end{array}$

